\title{
What is typical language development?
}

Michael S. C. Thomas, Themelis Karaminis, \& Victoria C. P. Knowland

Developmental Neurocognition Lab, Centre for Brain and Cognitive Development,

Birkbeck College, University of London, UK.

Running head: What is typical language development?

Word count: 3200 main text

Address for correspondence:

Dr. Michael Thomas

Developmental Neurocognition Lab

Department of Psychological Sciences

Birkbeck College

Malet Street

London WC1E 7HX, UK

Email: m.thomas@bbk.ac.uk

Web: http://www.psyc.bbk.ac.uk/research/DNL/

Tel.: +44 (0)20 76316386

Fax: +44 (0)20 76316312 


\begin{abstract}
In light of Musolino, Chunyo and Landau's findings and conclusions regarding syntax comprehension in Williams syndrome (this issue), we review the criteria used to determine whether the development of language is typical or atypical and our current understanding of the causes of language delay. Given a certain set of theoretical assumptions (e.g., generative / modular), fairly poor performance can nevertheless be viewed as indicating typical development. Given other theoretical assumptions (e.g., a neuroconstructivist view of constrained development), the same data can be viewed as indicative of atypicality.
\end{abstract}




\section{Introduction}

A group of individuals with the rare genetic syndrome Williams syndrome (WS), who have a mean chronological age of sixteen years, are given a language comprehension task. The task has greatly simplified task demands: the individuals need only decide whether a spoken sentence is a correct depiction of a pictorially represented scenario: yes or no. The individuals with WS perform much worse on this task than would be expected for their age (in this particular study, age appropriate performance is estimated from that of undergraduate students). Indeed, the individuals with WS perform so poorly that they are worse than six-year-old children, some ten years younger. The task also includes control conditions, which are used to predict performance on the key experimental conditions. The individuals with WS show a different relationship between control and experimental conditions to the six year olds. They do, however, show a similar relationship as that observed in four-year-old children (some twelve years younger), while performing at a higher overall level than the four year olds.

From the behavioural data of this sort, Musolino, Chunyo and Landau (this issue) concluded that individuals with WS acquire language no differently and develop grammars indistinguishable from those of typically developing individuals. They concluded that language acquisition is not fundamentally altered in WS.

How did we get to a point where such poor performance on a language comprehension task can be viewed as evidence of normal processes of language development? If such poor performance can be viewed as evidence of normality, what does it take not to have normally developing language according to this task, or more generally? In this article, we consider the theoretical assumptions necessary to draw conclusions like 
those of Musolino, Chunyo and Landau (henceforth MCL) from the observed behavioural data, and place them in the context of alternative theoretical assumptions that would lead to a different conclusion. We take the opportunity to review our current understanding of the notion of developmental delay, and to consider the criteria that determine when poor language performance in developmental disorders should be viewed as atypical development.

\section{Assumptions}

First, some background to this debate: MCL favour a generative / modular view of the development of the language system and in respect of WS, the hypothesis that the computational system of language (abstract grammatical knowledge) develops normally in this disorder despite moderate learning disability. MCL cast this view in opposition to one particular neuroconstructivist hypothesis on language development in WS (the Imbalance hypothesis; Thomas \& Karmiloff-Smith, 2003). MCL interpreted the Imbalance hypothesis as (a) rejecting modularity as a developmental startstate and (b) proposing that language develops atypically in WS. The behavioural data were then offered as favouring the modular view and sufficient to reject the neuroconstructivist Imbalance hypothesis.

Notably, MCL's argument did not proceed by direct falsification. Their study could have been constructed in the following way: Theoretical Position 1 predicts data A, Theoretical Position 2 predicts data B; the results turned out to be data A, therefore Position 1 was supported and Position 2 was falsified. In contrast, the behavioural data had aspects that could be viewed by both Position 1 and Position 2 as supporting their theories, depending on the assumptions used in interpreting the results. The MCL study, therefore, 
revolved around a particular way of interpreting the data, rather than arbitrating between hypotheses from competing theories.

What are the assumptions by which MCL were able to view WS language development as normal despite the low levels of accuracy exhibited by the group? There are two. The first assumption is that in the comprehension task, above chance accuracy levels under binary-forced-choice conditions can only be achieved by possession of certain key syntactic and semantic knowledge. Any performance above chance is then deemed sufficient to demonstrate the presence of this knowledge. The second assumption relies on a distinction employed in the generative tradition between syntactic knowledge (competence) and the processes making use of that knowledge (performance). The second assumption is that the extent to which accuracy exceeds chance levels (for instance compared to chronological or mental-age-matched controls) reflects performance factors only, and not differences in underlying grammatical knowledge. With these assumptions in place, once above-chance accuracy levels were observed, the authors could conclude that individuals with WS have normal grammatical knowledge. Note that the second assumption is not necessarily reflective of the generative approach per se. For example, Perovic and Wexler (in press) recently used a binary-forced-choice sentence-picture matching task to explore comprehension of the verbal passive in children with WS. Perovic and Wexler interpreted accuracy levels that differed reliably from chance in the disorder group as indicative of atypical syntactic knowledge (specifically, a difficulty forming argument chains) based on comparison to several control groups matched on standardised measures including non-verbal reasoning, receptive vocabulary, and receptive grammar. 
Like Perovic and Wexler, a neuroconstructivist would view differences in accuracy levels above chance compared to control groups as informative about the developmental state of the language comprehension system. A comparison of the developmental state of this system to other components of the overall language system (e.g., phonology, vocabulary, pragmatics) would be taken as informative about the developmental history of the language system and the nature of the constraints operating on it. How was task performance related to other language skills? MCL compared the WS group to a control group matched on the non-verbal sub-test of the Kaufman Brief Intelligence Test (KBIT; Kaufman \& Kaufman, 1990). The results indicated that comprehension in the syntax/semantics task was poorer in the WS group than expected given their non-verbal intelligence. MCL reported that the individuals with WS performed better on the verbal sub-test of the KBIT, which tapped receptive vocabulary ability, than the non-verbal subtest of the KBIT. We can therefore also infer that WS performance on the syntax/semantics task was (much) poorer than expected given their receptive vocabulary ability.

For the neuroconstructivist, three aspects of the empirical data might be viewed as clues that the language system is not developing normally: (1) the presence of differences in performance levels compared to chronological and mental age matched control groups; (2) an unusual developmental relationship between the experimental and control conditions within the task; and, (3) an unusual developmental relationship between performance on the syntax/semantics task and receptive vocabulary ability. Moreover, because neuroconstructivism does not embrace the distinction between competence and performance, a primary focus is why there should be such a large developmental delay in 
language comprehension - even if to answer this question involves understanding the nature of learning disability itself.

In short, based on the behavioural data reported by MCP, the description of WS syntax development as normal depends on a set of a priori assumptions about which aspects of the behavioural data are of interest.

\section{Implications for modularity}

In their discussion, MCL considered more widely whether their data on language comprehension in WS pose a challenge to neuroconstructivism. They accepted some of the tenets of neuroconstructivism (the importance of adopting a developmental perspective; ultimately seeking for lower-level underlying causes for developmental disorders). However, they maintained generally that modularity should be a central concept in explaining the uneven cognitive profile observed in WS, and specifically that the normal development of syntax (as they construed it) represents a capability that develops normally independently from the rest of the language system and independently from the rest of cognition.

Two points are worth making here. First, an additional tenet of neuroconstructivism is the importance of the sensitivity of the behavioural measures in determining whether a given ability develops normally (Karmiloff-Smith, 1998; Mareschal et al., 2007). It has already been demonstrated that if insensitive behavioural measures are used (e.g., those relying on accuracy, without time pressure, and with restricted options for responding), then it is possible for atypical cognitive processes to deliver behaviours that fall in the normal range. Indeed, we know this to be true of WS itself, in the domain of face recognition: for their age-appropriate level of face recognition on a relatively insensitive 
standardised test (the Benton task; Benton, 1983), children with WS revealed atypical underlying processes on more sensitive experimental measures (Annaz et al., 2009; Karmiloff-Smith et al., 2004). One important question, then, is whether the Truth Value Judgement Task (Crain \& Thornton, 1998) used by MCL is a sufficiently sensitive measure to tell us how children were producing their responses on the task. Each of the conditions used by MCL required only eight binary forced-choice decisions. Responses were not speeded and the nature of incorrect responses was uninformative. As Leonard (1998) has argued, one of the key markers of atypical rather than delayed development is qualitatively atypical errors in the disorder group (see also Thomas et al., 2009). Yet the task design offered no scope for such errors and therefore reduced the opportunity to classify development as anything other than delayed. Given the limited information provided by the task measure, MCL had to assume that task performance necessarily reflected the requisite knowledge of core syntactic and semantic principles posited in their theoretical framework. Of course, in doing so, MCL were right to point out that any competing hypothesis must explain how else above-chance performance could be achieved on their task.

The second point worth making is that the neuroconstructivist emphasis on developmental change has implications for the role that modularity can play in explaining uneven cognitive profiles. The emphasis on developmental change leads both to the use of particular methodological approaches (e.g., research designs that trace developmental trajectories, rather than collapsing participant groups over wide age ranges; see Thomas et al., 2009), and to the formulation of explanations with certain characteristics (e.g., theories that features concepts such as plasticity, adaptation, interactivity, redundancy, and compensation; see Thomas, 2005). Within a developmental framework, it is far from clear 
that invoking modularity per se in the startstate of a given disorder is sufficient to explain proposed selective deficits or islets of normality in behaviours observed in later childhood (Thomas, Purser \& Richardson, in press). Since there must be communication between modules to deliver functionality (whatever the commitment to encapsulation of knowledge within modules), why shouldn't such communication lead to a spread of deficits throughout communicating modules? Or, conversely, allow a deficit to be compensated for amongst a set of modules? The computational principles that would or would not permit deficit spread or deficit compensation have been a central concern of neuroconstructivists (see e.g., Baughman \& Thomas, 2008; Thomas \& Karmiloff-Smith, 2002) because to identify these principles is to identify the constraints that shape development.

Contra MCL, then, we argue that modularity is not the key concern; the key concern is specifying the nature of the developmental process.

\section{A modular neuroconstructivist approach?}

While MCL took their findings to falsify one neuroconstructivist hypothesis of language development in WS (the Imbalance Hypothesis), they took them to favour another (the Conservative Hypothesis). MCL argued, however, that the Conservative Hypothesis is in fact a species of modular account. Under the Conservative Hypothesis, language development in WS is held to be line with non-verbal mental age, once non-verbal intelligence tests that depend on visuo-spatial skills have been stripped out - since these are a known weakness in the disorder (Brock, 2007; Thomas, 2008; Thomas \& KarmiloffSmith, 2003; see also Mervis, 2006, for the view that language in WS is normal but 
delayed.) If language development is simply delayed then, argued MCL, it must have the same modular structure. ${ }^{1}$

It is certainly true that the Conservative Hypothesis implies that many of the wider constraints shaping language development in WS are not perturbed by the disorder: for example, basic input-output systems and channels of information flow such as the motor systems driving articulation, the perceptual systems interpreting input, the multi-modal systems linking to conceptual knowledge, and pragmatic systems linking with social and emotional systems. Moreover, the mechanisms that process sequential structure in production and comprehension would have to bear some relation to those found in the range of normal development. However in WS, all these systems appear to deliver performance that is lower than expected given chronological age. And the empirical data remain mixed on whether the state of the component systems in WS is identical to that found in younger typically developing children. For example, data do exist that indicate anomalies in semantics, pragmatics, and syntax (e.g., respectively, Thomas et al., 2006; Bishop \& Laws, 2004; Perovic \& Wexler, in press). The fact that phonology appears to be one of the strongest domains in WS may underlie the prominence of receptive vocabulary skills, as well as the relative strength in language overall compared to disorders characterised by phonological deficits (such as Down syndrome and Specific Language Impairment). To argue that phonology explains superior language development compared to some other disorders is not the same as arguing that all WS language is achieved by rote

\footnotetext{
${ }^{1}$ Strictly speaking, MCL's data are only partially consistent with the Conservative Hypothesis. They are consistent because, within the authors' framework, they observed no markers of atypicality. They are inconsistent because the results indicated WS task performance was in fact worse than would be expected given their non-verbal mental age.
} 
memorisation of phonological forms. McDonald (1997), for instance, argued that the quality of phonological representations is a predictor of the relative success or failure of language acquisition across a range of typical and atypical populations, including early and late first and second language learners.

\section{Delay - of no theoretical interest?}

We have seen that MCL felt able to characterise language as developing normally in WS despite a developmental delay of more than 10 years on their task. The implication was that delay does not bear on the normality of development. But what is the explanation for the delay? Presumably, one could argue that in the case of syntax development, the relevant mechanisms are afflicted by whatever it is that causes learning disability in the rest of the system; or that mechanisms on which syntax acquisition relies such as vocabulary and working memory are so afflicted.

This line of argument glosses over the fact that we have little idea of what delay actually constitutes, other than a re-description of the behavioural observation that performance resembles that of younger typically developing controls. Descriptively, delay is a blunt term that conflates several different patterns of divergence from the range of typical developmental trajectories (Thomas et al., 2009). Mechanistically, the situation is worse. They are no concrete proposals of how delay should work. Were we to formulate a mechanistic account, testable predictions should surely follow. For example, if one thought that the mechanisms mediating development just worked more slowly, one might predict some of the following: (1) for any domain with a ceiling performance level, individuals with delay should eventually catch up; (2) in those sensory domains with sensitive periods, the periods should be extended (for example, in specialisation to the phonemic contrasts of 
one's own language); (3) in domains where there is specialisation of function, this specialisation should also emerge later (e.g., in face recognition, for faces in an upright orientation); and (4) there should be identical quality of processing when individuals are matched for performance level, where quality is assessed by the effect of implicit variables like frequency, imageability, similarity, and so forth. (5) Where delay is widespread, the reduction in rate should be the same across all cognitive domains, since the same mechanism can't obviously explain lots of different delays (other than posthoc). (6) Where delay is argued to be focal, under no developmental theory should any other cognitive system rely on the affected component for its own successful development. Hypotheses such as these are rarely articulated, let alone tested (and in some cases, they are obviously false - individuals with delay rarely reach full adult levels of performance). And this is indicative of the very provisional status of 'delay' as an explanatory concept.

Indeed, by considering the 'causes of delay', we many not even be asking the right question. The deeper issue here is what causes developmental profiles in a given cognitive domain to be similar and what causes them to be different. Some researchers are inclined to view similarities as solely arising from internal constraints or mechanisms (see Thomas, 2005, for discussion). But there are at least two other potential sources: the structure of the cognitive domain (in terms of which aspects are hard and which aspects are easy, a factor that will hold across a range of architectures attempting to acquire the domain), and the information available in an individual's subjective environment. For example, recent research on the impact of socio-economic status (SES) on syntax development revealed similarities across SES groups on measures tapping mastery of basic syntactic rules of simple sentences, but differences in the mastery of complex sentence structures that were 
apparent from the earliest stages of production of multi-clause sentences (Vasilyeva, Waterfall \& Huttenlocher, 2008). In this case, both similarities and differences emerge from an environmental variable.

It is therefore far from clear that we should dismiss delay as irrelevant to whether language development is typical or not. When we allude to the phenomenon of delay, we merely highlight those aspects of developmental profiles that are qualitatively similar between groups when chronological age is ignored, without requiring deeper insights into the reason why. One method to pursue such insights is by explicit implementation of the developmental process in computational models (see, e.g., Thomas, Ronald \& Forrester, submitted). Here it becomes apparent that minimally, delay may result from attempting to solve roughly the same problem, with poorer computational resources or from using the same computational resources to solve a problem when provided with poorer information.

So far we have considered delay in respect of individual domains. How does one characterise a disorder if all aspects of language (or cognition) are delayed, but the size of the delay differs across these components? A focus on any one domain indicates performance resembles that of younger typically developing controls. If delay is of no theoretical interest, one would conclude that all the components of the system are developing normally, just with different delays. Nevertheless, the component parts of the cognitive system must interact with each other to deliver function and in many cases, to develop future functions. How could the delays be different? To put it more starkly, isn't differential delay across the components of the cognitive system itself atypical? 


\section{Conclusion: The nature of syntax in WS}

The strength of MCL's study of language comprehension in WS is to challenge those researchers who claim WS language development is atypical to explain how the degree of successful behaviour that was observed (in this case, above chance performance in the Truth Value Judgement Task) could have been produced using different underlying processes. Such an account might presumably appeal to lexical or semantic/pragmatic compensatory mechanisms, or comprise processes that contain some but not all of the grammatical properties outlined in the generative theory, or employ computational mechanisms that approximate formal syntactic systems under some processing conditions but not others (Christiansen \& Chater, 2001; Rumelhart \& McClelland, 1986). The conclusion that the results are evidence of normal language development in the disorder are undermined by the low accuracy levels exhibited by the individuals with WS (worse indeed than their level of non-verbal cognition) and the presence of at least one marker of atypicality, namely the relationship between component language skills. We have argued that the authors' conclusion is partly dependent on a set of assumptions regarding how the experimental data should be interpreted. Nevertheless, even with similar assumptions and methods, other researchers have recently come to the opposite conclusion, that aspects of syntax develop atypically in WS. Perovic and Wexler (in press) found deficits in the comprehension of the passive and interpreted their evidence as pointing to a dissociation between aspects of linguistic knowledge in the disorder. A juxtaposition of the respective findings of Musolino, Chunyo and Landau and Perovic and Wexler indicate that there is still work to be done before a consensus emerges on the nature of linguistic knowledge in Williams syndrome and its developmental origins. 


\section{References}

Annaz, D., Karmiloff-Smith, A., Johnson, M. H., \& Thomas, M. S. C. (2009). A crosssyndrome study of the development of holistic face recognition in children with autism, Down syndrome and Williams syndrome. Journal of Experimental Child Psychology, 102, 456-486.

Baughman, F. D. \& Thomas, M. S. C. (2008). Specific impairments in cognitive development: A dynamical systems approach. In B. C. Love, K. McRae, \& V. M. Sloutsky (Eds.), Proceedings of the 30th Annual Conference of the Cognitive Science Society (pp. 1819-1824). Austin, TX: Cognitive Science Society

Benton, A., Hamsher, K., Varney, N. R., \& Spreen, O. (1983). Benton Test of Facial Recognition. New York: Oxford University Press.

Brock, J. (2007). Language abilities in Williams syndrome: A critical review. Development and Psychopathology, 19, 97-127.

Crain, S., \& Thornton, R. (1998). Investigations in universal grammar. Cambridge, MA: MIT Press.

Christiansen, M. H., \& Chater, N. (2001). Connectionist psycholinguistics. Westport, CT: Ablex Publishing.

Karmiloff-Smith, A. (1998). Development itself is the key to understanding developmental disorders. Trends in Cognitive Sciences, 2, 389-398.

Karmiloff-Smith, A., Thomas, M., Annaz, D., Humphreys, K., Ewing, S., Brace, N., Van Duuren, M., Pike, G., Grice, S., \& Campbell, R. (2004). Exploring the Williams syndrome face-processing debate: the importance of building developmental trajectories. Journal of Child Psychology and Psychiatry, 45(7), 1258-1274 
Kaufman, A. S., \& Kaufman, N. L. (1990). Kaufman brief intelligence test. Circle Pines, MN: AGS Publishing.

Laws, G., \& Bishop, D. (2004). Pragmatic language impairment and social deficits in Williams syndrome: a comparison with Down's syndrome and specific language impairment. International Journal of Language Communication Disorders, 39(1), 4564.

Leonard, L. (1998). Children with Specific Language Impairment. Cambridge, MA: MIT Press. Mareschal, D., Johnson, M., Sirios, S., Spratling, M., Thomas, M. S. C., \& Westermann, G. (2007). Neuroconstructivism: How the brain constructs cognition. Oxford: Oxford University Press.

McDonald, J. L. (1997). Language acquisition: The acquisition of linguistic structure in normal and special populations. Annual Review of Psychology, 48, 215-241.

Mervis, C. B. (2006). Language abilities in Williams-Beuren syndrome. In C. Morris, H. Lenhoff, and P. Wang (Eds.), Williams-Beuren syndrome: Research, evaluation, and treatment. Baltimore: The Johns Hopkins University Press.

Musolino, J., Chunyo, G., \& Landau, B. (2010). Uncovering knowledge of core syntactic and semantic principles in individuals with Williams syndrome. Language Learning and Development.

Perovic, A., \& Wexler, K. (in press). Development of verbal passive in Williams syndrome. Journal of Speech, Language, and Hearing Research.

Thomas, M. S. C. (2005). Constraints on language development: Insights from developmental disorders. In P. Fletcher \& J. Miller (Eds.) Language Disorders and Developmental Theory. John Benjamins. 
Thomas, M. S. C. (2008). L'acquisition du langage dans les pathologies du diveloppement [Language development in developmental disorders]. In M. Kail, M. Fayol, \& M. Hickmann, L'apprentissage des langues, (pp. 451-475). Paris: CNRS Editions.

Thomas, M. S. C., Annaz, D., Ansari, D., Serif, G., Jarrold, C., \& Karmiloff-Smith, A. (2009). Using developmental trajectories to understand developmental disorders. Journal of Speech, Language, and Hearing Research, 52, 336-358.

Thomas, M. S. C., Dockrell, J. E., Messer, D., Parmigiani, C., Ansari, D., \& KarmiloffSmith, A. (2006). Speeded naming, frequency and the development of the lexicon in Williams syndrome. Language and Cognitive Processes, 21(6), 721-759.

Thomas, M. S. C. \& Karmiloff-Smith, A. (2002). Are developmental disorders like cases of adult brain damage? Implications from connectionist modelling. Behavioral and Brain Sciences, 25(6), 727-788.

Thomas, M. S. C. \& Karmiloff-Smith, A. (2003). Modelling language acquisition in atypical phenotypes. Psychological Review, 110(4), 647-682.

Thomas, M. S. C., Purser, H. R. M., \& Richardson, F. M. (in press). Modularity and developmental disorders. In: P. D. Zelazo (Ed), Oxford Handbook of Developmental Psychology. Oxford: Oxford University Press.

Thomas, M. S. C., Ronald, A., \& Forrester, N. A. (submitted). Modelling the mechanisms underlying population variability across development: Simulating genetic and environmental effects on cognition. Manuscript submitted for publication.

Vasilyeva, M., Waterfall, H., \& Huttenlocher, J. (2008). Emergence of syntax: commonalities and differences. Developmental Science, 11(1), 84-97. 


\section{Acknowledgements}

This research was supported by MRC Career Establishment Grant G0300188, British Academy grants LRG-35369 and SG-040400, EC grant 0209088 (NEST), an ESRC student. The research of second author was supported by the Greek State Scholarship Foundation (IKY). The research of the third author was supported by a UK ESRC studentship. 